\title{
Reduction of Acromioclavicular Joint Type III Injuries and Coracoclavicular Ligament Reconstruction Using Semitendinosus Tendon Autograft and A Triple Suture-Cerclage
}

\author{
Mohammed M. Mansour, MD, Mohamed A. Abdelsalam, MD \\ Orthopaedic Department, Faculty of Medicine, Zagazig University, Egypt \\ yousufmmkh@gmail.com
}

Received Date: July 10, 2017

Accepted Date: July 17, 2017

Published Date: July 28, 2017

Abstract

Background: The management of acute Rockwood type III Acromioclavicular joint (ACJ) dislocation remains controversial. Good-to-excellent results can be obtained with nonsurgical management, however, surgical management for type III acute ACJ dislocation restores joint anatomy, thus avoiding obvious deformity, persistent pain and residual symptoms.

Methods: In this prospective studynineteen (14 men, 5 women) patients, withRockwood type III (ACJ) dislocation, underwent coracoclavicular (CC)ligament reconstructive surgery using a semitendinosus autograft and a triple non-absorbable suture-cerclage. The mean patient age was 33.1 years (range, 19-46). All patients were evaluated clinically preoperativeand at final follow-up usingVisual Analogue Scale,Constant Shoulder Score and DASH scoreand evaluatedradiologicallypreoperative, on the second day postoperative and at final follow-upusingCoracoclavicular distance (CCD) difference to the unaffected side.The mean follow-up was 25 months (range, 18-37).

Results: At the final follow-up, the Visual Analogue Scale (mean 0.7) revealed a good to excellent clinical outcome. The mean Constant-Murley score was 95.2 (range 77-100). The DASH score (mean 3.52 points). The difference in the CCD compared to the contralateral side was $<5 \mathrm{~mm}$ for 17 patients, between $5-10 \mathrm{~mm}$ for 2 patients. In the anteroposterior (AP) plane, 19 (100\%) of the patients maintained complete reduction.

Conclusion: Acromioclavicular joint reduction with reconstruction of the coracoclavicular ligament using semitendinosus tendon autograft and a triple non-absorbable suture-cerclageappears to be a good means with satisfactory clinical and radiological results for treating acute Rockwood type II Acromioclavicular dislocation.

Keywords: acromioclavicular joint; dislocation; coracoclavicular ligament; reconstruction.

\section{INTRODUCTION}

AC jointinjuries account for about $12 \%$ of all shoulder injuries inclinical practiceand nearly $50 \%$ in athletes participatingin contact sports [1].

Suspicion of AC joint injury should prompt the clinician to obtain standard AP, axillary, scapular Y, and Zanca films which are AP film with 10-15 degrees cephalad tilt to removes superimposing structures and allows complete visualization of the clavicle and its corresponding articulations. Inclusion of both clavicles on one radiograph is also helpful for comparison to the uninjured side. An axillary view is obtained to evaluate the humeral head, glenoid surface, and degree of a potential posterior displacement of the clavicle (as in Rockwoodtype IV injuries) [2]. 
Reduction of Acromioclavicular Joint Type III Injuries and Coracoclavicular Ligament Reconstruction Using Semitendinosus Tendon Autograft and A Triple Suture-Cerclage

Although there is agreement about conservative treatment for types I and II AC joint dislocations [3], and surgical treatment for types IV, V and VI AC joint dislocations [4].Type III is classified as a superior displacement of the lateral end of the clavicle of one clavicular diameter or $1 \mathrm{~cm}$ on the anteroposterior radiograph [5].

The management of acute Rockwood type III ACJ dislocation remains controversial. Some authors obtained good-to-excellent results with nonsurgical management [6]; however, others have reported that Surgical management fortype III acuteACJ dislocationrestores joint anatomy, thus avoiding obvious deformity, persistent pain and residual symptoms associated with the acromioclavicular joint at final follow-up evaluations and is typically recommended for high functioning individuals [7-8].

Rolf et al.[9] found that patients with type III injuries who undergo early CC ligament reconstruction have significantly improved outcomes when compared with those who underwent a trial of therapy followed by surgical treatment greater than 3 months from the index injury.

Many operative techniques have been used for treatment of AC joint dislocation. These can be divided into 2 groups focusing on the ligament healing or on the ligament reconstruction. The first techniques try to maintain the clavicle-coracoid relationship in a reduced position in order to allow primary healing of the CC ligaments, but they are not indicated for chronic dislocations. The second group techniques focuses on CC ligament reconstruction. They are indicated in high-grade dislocations or in chronic injuries [10].

AC-joint stabilization can be performedthrough open or arthroscopically assistedtechniques. Several open and arthroscopic techniques havebeen described for the treatmentof AC joint dislocation [11].

\section{Patients}

Nineteen (14 men, 5 women) patients, with Rockwood type III ACJ dislocation, underwent CC ligament reconstructive surgery using a semitendinosus autograft and a triple non-absorbable suture-cerclage. The mean patient age was 33.1 years (range, 19-46). All patients were evaluated clinically preoperative and at final follow-up using Visual Analogue Scale,Constant Shoulder Score and DASH score and evaluated radiologically preoperative, on the second day postoperative and at final follow-up using Coracoclavicular distance (CCD) difference to the unaffected side. The mean follow-up was 25 months (range, 18-37).

Table1. Demographic Data of the Patients

\begin{tabular}{|l|l|}
\hline Number of patients & 19 \\
\hline Mean age (range) & 33.1 years (range, 19-46) \\
\hline Gender N (\%) & 14 men, 5 women \\
\hline Dominant shoulder $\boldsymbol{N}(\%)$ & $12(63.1 \%)$ \\
\hline Mean time between injury and surgery, days (range) & $14(5-21)$ \\
\hline Mean duration of surgery, minutes (range) & $88(80-105)$ \\
\hline
\end{tabular}

SURGICAL TECHNIQUE

Patient weredone under general anesthesia and placed in a beach chair position.Semitendinosus tendon autograft from the ipsilateral side was harvested and prepared in the standard technique. An anterior approach using a vertical skin (saber-cut) incision medial to the $\mathrm{AC}$-joint spanning from the clavicle to just inferior to the coracoids process (fig. $1 \mathrm{E}$ ). The supraclavicular nerves were identified and protected throughout the procedure. The trapezius deltoid fascia was opened transversely. The deltoid was dissected off the anterosuperior clavicle subperiosteally, allowing visualization of the coracoid process and the acromioclavicular joint(fig. 1 A). A deltoid split can be performed in line with its fibers if additional exposure is needed. Care was taken not to 
Reduction of Acromioclavicular Joint Type III Injuries and Coracoclavicular Ligament Reconstruction Using Semitendinosus Tendon Autograft and A Triple Suture-Cerclage

damage the coracoacromial ligament when dissecting the area between the clavicle and the coracoid. The trapezoid component of the coracoclavicular ligament spans from the superior surface of the posterior half of the coracoid process. The conoid component is attached to the coracoid knucklejust posterior to the trapezoid attachment and runs cranially in an inverse cone shape to attach to the posterior margin of the clavicle at the junction of the middle and lateral one thirds. The medial and lateral borders of the coracoidprocess were identified. A 4.5-mm drill bit is positioned on the superior aspect of the clavicle andtwo holes are drilled with adequate bone bridge in between in a place tom mimic the anatomic position of the trapezoid and conoid parts of the coracoclavicualr ligaments (fig. $1 \mathrm{~B}$ ). After exposure of the coracoid process, a subcoracoid passage of the prepared semitendinosus autograft and triple non-absorbable suture was performed (fig. $1 \mathrm{C}$ ). Then the triple non-absorbable suture was passed through the lateral hole and the semitendinosus autograft was passed through the medial hole previously drilled in the clavicle. The torn disci in the AC- joint were removed. The ACJ was usually easily reduced as these were acute cases and reduction was maintained with direct pressure.The triple non-absorbable suture was tied at first to maintain the reduction and then the semitendinosus autograft was tied while the AC joint was well reduced to have the best tension of the ligament (fig. $1 \mathrm{D}$ ). Finally, the trapezoid/deltoid fascia was reconstructed and skin closed (fig. $1 \mathrm{E}$ ).

The shoulder was immobilized in a sling for six weeks. Passive and active (gravityassisted)exercises out of sling were started under physiotherapistssupervision two weeks after surgery limitingthe range of motion to $90^{\circ}$ of shoulder flexion andabduction. Six weeks after surgery, the sling wasremoved and full range of motion allowed. Heavy weightlifting and overhead sporting activities were restrictedfor 12 weeks postoperatively.

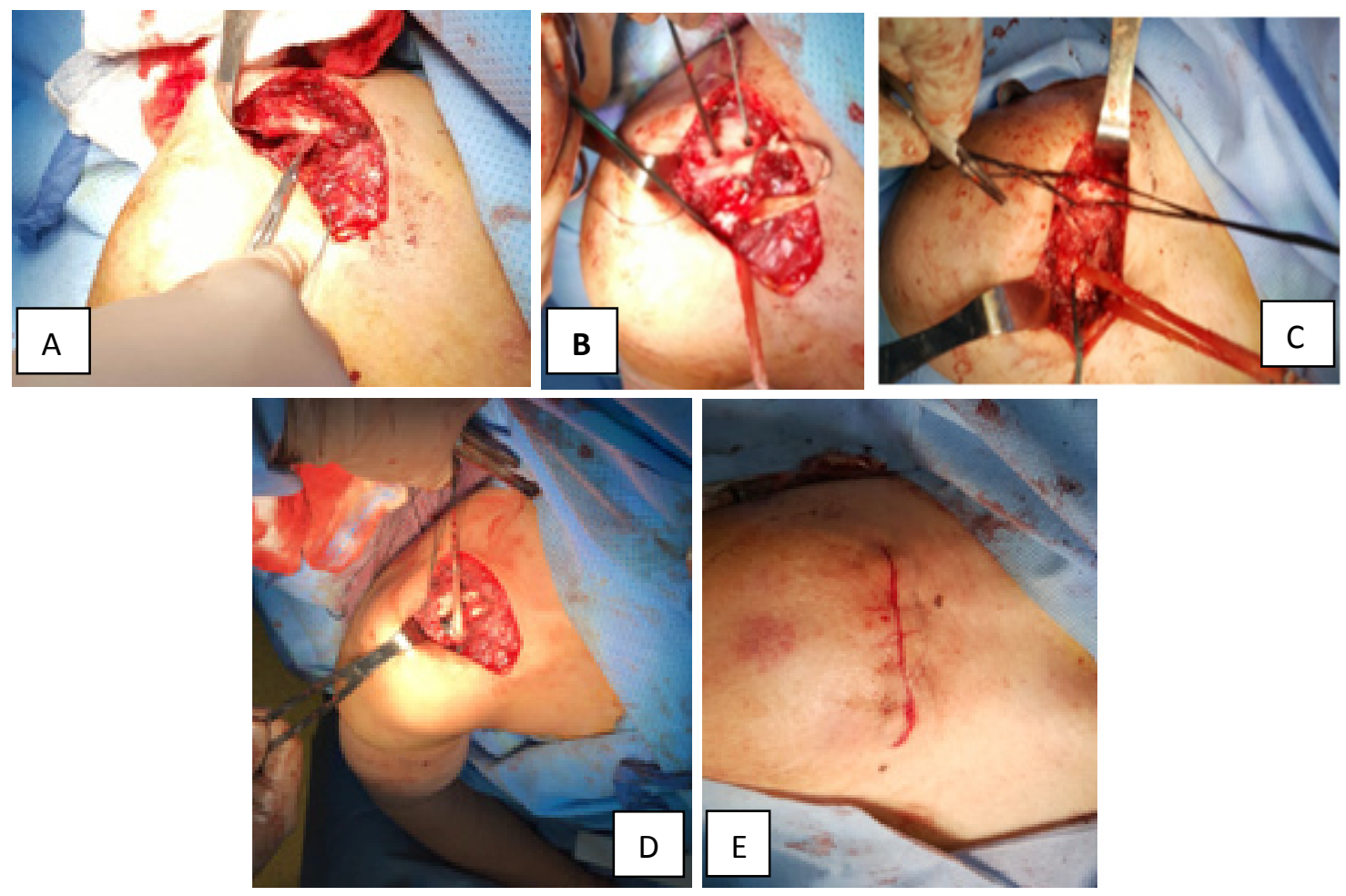

Figure1. Surgical technique: $\boldsymbol{A}$, the ACJ is dislocated with disruption of the CC ligament. $\boldsymbol{B}$, two holes drilled in the clavicle. $\boldsymbol{C}$, the semitendinosus autograft and the triple suture after subcoracoid passage. $\boldsymbol{D}$, the triple suture and the semitendinosus autograft are tied after being passed through the drill holes. $\boldsymbol{E}$, after closure of the wound 
Reduction of Acromioclavicular Joint Type III Injuries and Coracoclavicular Ligament Reconstruction Using Semitendinosus Tendon Autograft and A Triple Suture-Cerclage

\section{RESULTS}

All patients were available forfollow up and were evaluated clinically and radiologically.The mean duration of follow up was25 months (range, 18-37), and the mean patient age was 33.1 years (range, 19-46). All patients were evaluated preoperative on the second day postoperative and at final follow-up.

Clinical outcome: at the final follow-upthe Visual Analogue Scale (mean 0.7) revealed a good to excellent clinical outcome.The mean Constant-Murley [12] scorewas 95.2 (range 77-100). The DASH score (mean 3.52 points) and. Clinical examination showed no limitation of the rangeof motion in abduction, flexion, external and internalrotation compared to the contralateral shoulder. One patient (5.2\%) suffered superficial infection and was treated with antibiotic.

Radiological outcome: the pre-operative, the postoperativeand the x-rays obtained at final follow-upwere reviewed (fig 2 \&3).A significant difference was found in the CoracoClavicular Distance (CCD) between pre-operative and post-operative radiographs onsecond day after surgery (mean $18.7 \mathrm{~mm}$ versus $9.3 \mathrm{~mm}$. At time of final follow-up, the CCD had significantlyincreased to $11.2 \mathrm{~mm}$ in one patient, comparedto his post-operative value $9.6 \mathrm{~mm}$ and to the CCD ofthe contralateral uninjured shoulder $9.8 \mathrm{~mm}$.

The CCD difference compared to the contralateral side was divided into three groups: $<5 \mathrm{~mm}, 5-10 \mathrm{~mm}$ and $>10$ $\mathrm{mm}$. There were 17 patients (89.4\%) with a distance $<5 \mathrm{~mm}, 2$ patients with a distance of $5-10 \mathrm{~mm}(10.6 \%)$.

There was no statistical correlation between the clinicaloutcome (measured by the Constant score) and theradiological outcome (CC- difference to the unaffectedside). There were no fractures of the coracoid process in the study patients.
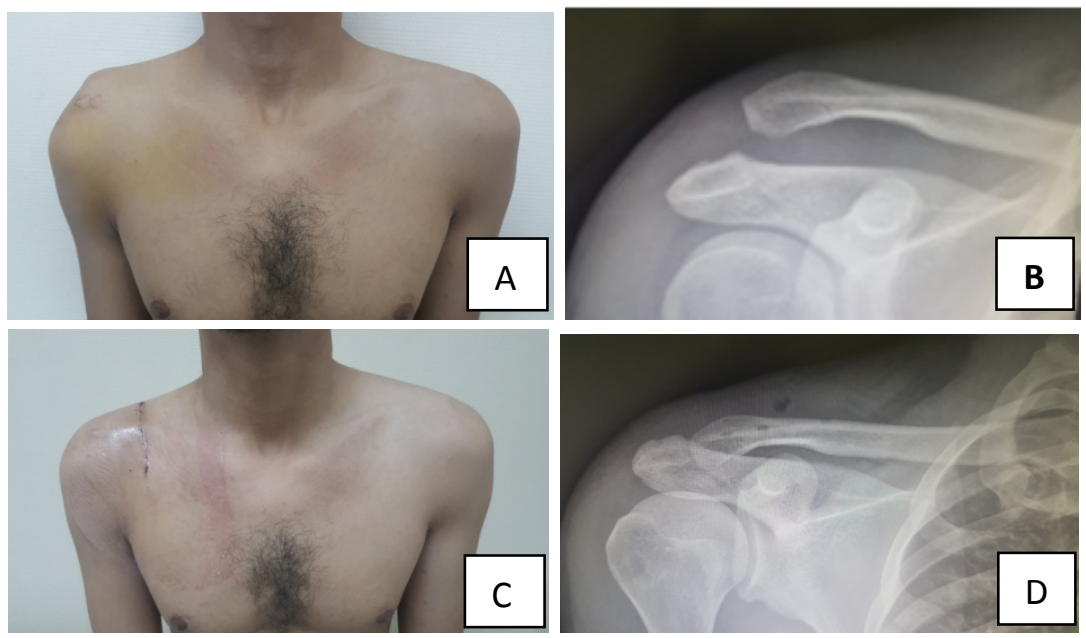

Figure2. $A \& B$, Preoperative photo and x ray $C \& D$, postoperative photo and x ray of type III AC dislocation
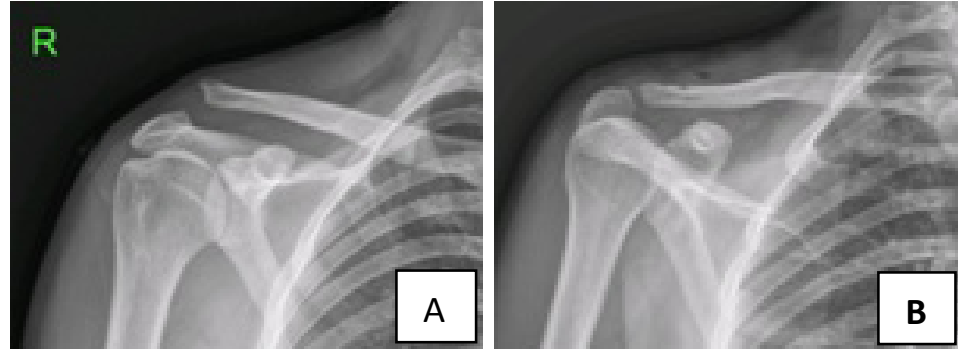

Figure3. $\boldsymbol{A}$, Preoperative x ray of type III AC dislocation $\boldsymbol{B}$, postoperative x ray 
Reduction of Acromioclavicular Joint Type III Injuries and Coracoclavicular Ligament Reconstruction Using Semitendinosus Tendon Autograft and A Triple Suture-Cerclage

\section{DisCUSSION}

There is still no gold standard treatment forACJ type IIIdislocation. Better subjective and radiological resultswere observed after surgical reduction of ACJ type IIIdislocation in young active patients [13].

Although non-operative treatments are recommended for type III AC joint dislocations, results from recent studies have shown better functional outcomes for surgical treatments of this type of injury [14].

Stabilization of the AC joint is achieved by the joint capsule and the CC ligaments. A healthy AC joint has been shown to accommodate 4-6 $\mathrm{mm}$ of translation in the anterior, posterior, and superior planes under a $70 \mathrm{~N}$ load. Furthermore, the $\mathrm{AC}$ joint undergoes rotary motion of $5^{\circ}$ to $8^{\circ}$ during scapulothoracic motion and $40^{\circ}$ to $45^{\circ}$ during shoulder abduction and elevation. [15]

Lim [16] showed that the clavicle rises in full overhead elevation up to 35 degrees and rotates along its long axis by 45 degrees this explains why techniques using a rigid CC ligament fixation like the Bosworth screw [17], Kirschner wires, or Steinman pins have failed to achieve optimal results and fallen out of favor in clinical practice due to their high failure and complication rates, as the clavicle and the AC joint are highly flexible.

Anatomic reconstruction of the $\mathrm{CC}$ ligaments has taken place to allow for the natural movement of the clavicle using semi-rigid fixation techniques such as tendon grafts orcortical fixation button devices. [18]

In 2010, Salzmann et al. [19] reported outcomes of arthroscopic anatomic 2-bundle cortical fixation button reconstruction of acute AC joint separations. They showed satisfactory clinical results in 23 patients at a minimum of 24 months followup. However, an $11.5 \%$ (3/26) revision rate included 1 coracoid fracture, 1 button slippage, and 1 wound infection. Caudal migration of the button (4 patients) or break out (1 patient) was noted in $22 \%$, however, these radiographic findings did not negatively influence clinical outcomes.

Reconstructions that incorporate a biologic graft withsuture fixation (cortical fixation buttons and anchors) haveshown better results. DeBerardino et al. [20] showed excellentearly clinical results without complications after ACreconstruction using a hybrid device that incorporated a graft with suture fixation (GraftRope system, Arthrex Inc., Naples, FL), although concerns exist regarding coracoids fractures due to the size of the hole that needs to be drilled and the technical aspects of drilling such a hole in the base of the coracoid. Using a similar device.

Biomechanical data has demonstrated that free graft reconstruction techniques provide more stable fixation constructs than other techniques fir ACJ reduction and CC joint fixation[21].

Authors of this study believe that addition of a triple non-absorbable suture-cerclage would give better results and additional strength to the biological construct when used with semitendinosus autograft and can provide a better long term durability.

Distal clavicle excision followed by transfer of the coracoacromial (CA) ligament to the resected end of the distal clavicle was first described by Weaver and Dunn in 1972 [22]. However, the value of this technique has been questioned since failure rates as high as $30 \%$ have been reported [23].Grassbaugh et al., noted in their study the improvement in clinical outcomes and superior improvement of radiographic grade and CC distance for surgeries performed without distal clavicle resections and that all failures occurred in procedures performed with a distal clavicle resection [24].The distal clavicleplays an important role in stability of the AC joint andresection should be limited [25].

Lim [26] had a 50\% fixation failure rate when Tightrope fixation was used in primary procedures (excluding revision surgery). His retrieved specimen revealed multiple areas of abrasion on no. 5 Fibrewire suture. He 
Reduction of Acromioclavicular Joint Type III Injuries and Coracoclavicular Ligament Reconstruction Using Semitendinosus Tendon Autograft and A Triple Suture-Cerclage

postulated that the mechanism of failure is suture abrasion. This could have resulted from abrasion on metallic buttons, clavicle plate, or sharp bony edges of coracoid or clavicle.

\section{CONCLUSION}

Acromioclavicular joint reduction with reconstruction of the coracoclavicular ligament using semitendinosus tendon autograft and a triple non-absorbable suture-cerclage appears to be a good means with satisfactory clinical and radiological results for treating acute Rockwood type II Acromioclavicular dislocation.

\section{REFERENCES}

1. Simovitch R, Sanders B, Ozbaydar M, et al. Acromioclavicular joint injuries: Diagnosis and management. J Am Acad Orthop Surg. 2009;17:207-19.

2. Tischer T, Salzmann GM, El-Azab H, et al. Incidence of associated injuries with acute acromioclavicular joint dislocations types III through V. Am J Sports Med. 2009;37:136-9.

3. Hootman JM. Acromioclavicular dislocation: conservative or surgical therapy. J Athl Train. 2004;39(1):10-1.

4. Tauber M. Management of acute acromioclavicular joint dislocations: current concepts. Arch Orthop Trauma Surg. 2013;133(7):985-95.

5. Smith TO, Chester R, Pearse EO, Hing CB. Operative versus non-operative management following Rockwood grade III acromioclavicular separation: a meta-analysis of the current evidence base. J Orthop Traumatol. 2011;12:19-27.

6. Rawes ML, Dias JJ. Long-term results of conservative treatment for acromioclavicular dislocation. J Bone Joint Surg (Br). 1996;78(3):410-2.

7. Gstettner C, Tauber M, Hitzl W, Resch H. Rockwood type III Acromioclavicular dislocation: surgical versus conservative treatment. J Shoulder Elbow Surg. 2008;17(2):220-5.

8. Lizaur A, Sanz-Reig J, Gonzalez-Parreño S. Long-term results of the surgical treatment of type III acromioclavicular dislocations: an update of a previous report. J Bone Joint Surg (Br). 2011;93(8):1088-92.

9. Rolf O, Hann von Weyhern A, Ewers A, et al. Acromioclavicular dislocation Rockwood III-V: Results of early vs delayed surgical treatment. Arch Orthop Trauma Surg. 2008;128:1153-7

10. Spoliti M, De Cupis M, Via A.G., Oliva F. All arthroscopic stabilization of acute acromioclavicular joint dislocation with fiberwire and endobutton system. Muscles, Ligaments and Tendons Journal 2014; 3984 (4): 398-403

11. Baumgarten KM, Altchek DW, Cordasco FA. Arthroscopically assisted acromioclavicular joint reconstruction. Arthroscopy.2006;22:228.

12. Constant C. Murley AH. A clinical method of functional assessment of shoulder. Clin Orthop Relat Res 1987 ; 214 : 160-164.

13. De Carli A, Lanzetti R. M., Ciompi A, Lupariello D, Rota P and Ferretti A Acromioclavicular third degree dislocation: surgical treatment in acute cases. Journal of Orthopaedic Surgery and Research.2015;10:13

14. Gstettner C, Tauber M, Hitzl W, Resch H. Rockwood type III acromioclavicular dislocation: surgical versus conservative treatment. J Shoulder Elbow Surg. 2008; 17(2):220-5. 
Reduction of Acromioclavicular Joint Type III Injuries and Coracoclavicular Ligament Reconstruction Using Semitendinosus Tendon Autograft and A Triple Suture-Cerclage

15. Renfree KJ, Wright TW. Anatomy and biomechanics of the Acromioclavicular and sternoclavicular joints. Clin Sports Med. 2003;22:219-37.

16. Lim Y: Triple endobutton technique in acromioclavicular joint reduction and reconstruction. Ann Acad Med Singapore 2008, 37:294-299.

17. Bosworth BM: Acromioclavicular dislocation; end-results of screw suspension treatment. Ann Surg 1948, 127(1):98-111.

18. Milewski MD, Tompkins M, Giugale JM, et al. Complications related to anatomic reconstruction of the coracoclavicular ligaments. Am J Sports Med. 2012.

19. Salzmann GM, Walz L, Buchmann S, et al. Arthroscopically assisted 2-bundle anatomical reduction of acute Acromioclavicular joint separations. Am J Sports Med. 2010;38:1179-87.

20. DeBerardino, T. M., Pensak, M. J., Ferreira, J., et al. Arthroscopic stabilization of AC joint dislocation using the AC graftrope system. J. Shoulder Elbow Surg. 19:47-52, 2010.

21. Michlitsch MG, Adamson GJ, Pink M, et al. Biomechanical comparison of a modified Weaver-Dunn and a free-tissue graft reconstruction of the acromioclavicular joint complex. Am J SportsMed. 2010;38:1196-203.

22. Weaver JK, Dunn HK. Treatment of acromioclavicular injuries, especially complete acromioclavicular separation. J Bone Joint Surg Am. 1972;54:1187-94.

23. GrutterPW,PetersenSA.Anatomicalacromioclavicularligamentreconstruction:Abiomechanical comparison of reconstructive techniques of the acromioclavicular joint. Am J Sports Med. 2005;33:1723-8.

24. Grassbaugh J.A.,ColeC., Wohlrab K., and EichingerJ.Surgical Technique Affects Outcomesin Acromioclavicular Reconstruction. Journal of Surgical Orthopaedic Advances. 22,:71-76,2013

25. Corteen, D. P., Teitge, R. A. Stabilization of the clavicle after distal resection: a biomechanical study. Am. J. Sports Med. 33:61-67,2005.

26. Lim Y. W. Acromioclavicular Joint Reduction, Repair and Reconstruction Using Metallic Buttons Early Results and Complications. Techniques in Shoulder \& Elbow Surgery 8(4):213-221, 2007

Citation: Mohammed M. Mansour, MD, Mohamed A. Abdelsalam, MD. "Reduction of Acromioclavicular Joint Type III Injuries and Coracoclavicular Ligament Reconstruction Using Semitendinosus Tendon Autograft and A Triple Suture-Cerclage". American Research Journal of Orthopedics and Traumatology; V2, I1; pp: 1-7.

Copyright (C) 2017 Mohammed M. Mansour, MD, Mohamed A. Abdelsalam, MD. This is an open access article distributed under the Creative Commons Attribution License, which permits unrestricted use, distribution, and reproduction in any medium, provided the original work is properly cited. 